\title{
IUFOST2006/812 What's Special about Carotenoids?
}

\author{
G. Britton \\ University of Liverpool, School of Biological Sciences, Biosciences Building, Crown Street, L69 7ZB \\ Liverpool, United Kingdom \\ g.britton@liv.ac.uk
}

Carotenoids are familiar to us all because of their colour - think of, for example, oranges, tomatoes and carrots. Colour is a major criterion for selecting food: attractive bright colours are perceived as an indication of good quality and freshness. But carotenoids are also important micronutrients for human health. Beta-carotene and others have long been known as the main dietary source of vitamin A in most populations. Lutein and zeaxanthin are important in helping to prevent age-related macular degeneration, a major cause of loss or impairment of sight in the elderly. A carotenoid-rich diet is associated with reduced risks of major lifethreatening conditions such as cancer and coronary heart disease, and with beneficial effects on the immune response system. In this talk, the distribution of carotenoids in nature will be outlined briefly, with emphasis on identifying which foods provide the carotenoids that are considered most important as micronutrients. Much has been written about the benefits of carotenoids for health, but are these claims justified? By listening to what the chemical structure tells us about the special properties of carotenoids, we can better appreciate the particular difficulties associated with the absorption, transport and deposition of carotenoids. This approach also provides an essential background for evaluating and understanding how carotenoids may act in biological systems to provide the reported benefits. This introduction to the world of carotenoids will set the scene for the specialied presentations that follow. 